\title{
IMPROVEMENT OF RHIC WARM BEAM VACUUM FOR HIGH INTENSITY OPERATION*
}

\author{
P. He, H.C. Hseuh ${ }^{\dagger}$, R.C. Lee, M. Mapes, L.A. Smart, D. Weiss and S.Y. Zhang \\ Collider-Accelerator Department, BNL, Upton, NY 11973, USA
}

\begin{abstract}
With increasing ion beam intensity during recent RHIC operations, pressure rises of several decades were observed at a few warm vacuum sections. Improvement of the warm sections has been carried out in last year's shutdown. Extensive in-situ bakes, additional UHV pumping, electron detectors and beam tube solenoids have been implemented. Vacuum monitoring and interlock were enhanced to reduce premature beam aborts. The effectiveness of these measures in reducing the beam induced pressure bumps and in increasing the vacuum system reliability are discussed and summarized.
\end{abstract}

\section{INTRODUCTION}

RHIC has a circumference of $3.8 \mathrm{~km}$ and comprises two interweaving rings (named yellow ring and blue ring) that intersect with each other at six locations. The total length of warm sections is $\sim 1.4 \mathrm{~km}$, consisting of 24 insertion regions (single beam) at the ends of the long arcs, 12 final focusing regions between D0 and DX and six interaction regions (IP), as shown schematically in Fig. 1. The design vacuum of the warm sections is $<5 \times 10^{-10}$ Torr. The beamgas lifetime, dominated by nuclear scattering with cross sections of $\sim 10^{-24} \mathrm{~cm}^{2}$ for $\mathrm{Au}$, is several hundred hours at the design vacuum level, much longer than the ten-hour intra-beam scattering lifetime [1]. Background noise to the detectors, due to beam-gas events in warm sections bracketing the experimental detectors establishes the requirements of RHIC beam vacuum systems [2].

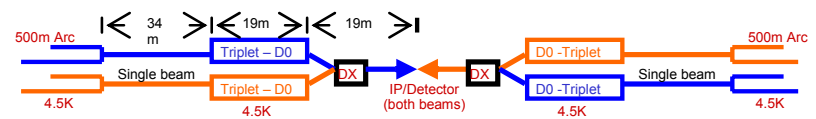

Fig. 1 RHIC warm sections bracketing the interaction points showing the incoming and outgoing single beam lines and the common lines at IP and DX-D0.

The warm sections are pumped by ion pumps (IP) and titanium sublimation pumps (TSP), and monitored with cold cathode gauges (CCG) at intervals averaging $14 \mathrm{~m}$. The pumping speed at the neck of the pumps is $\sim 500 \mathrm{l} / \mathrm{s}$ for active gases such as $\mathrm{CO}$ and $\mathrm{H}_{2}$. Due to the small linear conductance of the beam tubes, the effective pumping speeds are in the order of tens $1 / \mathrm{s} . \mathrm{m}$ for $\mathrm{H}_{2}$ and a few 1/s.m for CO. Most of the warm sections are in-situ bakeable up to $250^{\circ} \mathrm{C}$. The average pressures of the warm sections without beam have reached below the design vacuum level, owing to the gradual bakeout of these sections over the last three years.

No notable changes in pressure were observed during the 2000 beam commissioning run. However, pressure spikes of several decades occurred during the highintensity $\mathrm{Au} \times \mathrm{Au}, \mathrm{p} \times \mathrm{p}$ and $\mathrm{Au} \mathrm{x}$ d runs in the past three years. These rapid increases in pressure caused high detector background, sometimes exceeded the CCG set points for valve interlocks, and aborted the beams. The pressure rise was especially prominent during 110-bunch injection and became one of the major intensity-limiting factors for RHIC. Extensive bakeout of the warm sections and other improvement were carried out during 2002 summer shutdown to increase the intensity thresholds and to reduce the pressure rise. The effectiveness of these measures is summarized by comparing the beam and vacuum performance of $2003 \mathrm{Au} \times \mathrm{d}$ and $\mathrm{p} \times \mathrm{p}$ runs with those of $2002 \mathrm{p} \mathrm{x} \mathrm{p}$ and $2001 \mathrm{Au} \times \mathrm{Au}$ runs. Further improvement of the vacuum systems planned for the 2003 shut down is also described.

\section{VACUUM IMPROVEMENT}

\section{Pressure Rise vs In-Situ Bake}

To reduce detector background, the common beam regions bracketing the four major experiments were insitu baked before the 2001-2002 runs, and pressure of low $10^{-10}$ Torr and $10^{-11}$ Torr were achieved. Two types of pressure rise were observed $[3,4]$ during the 2001-2002 runs. The first type was the pressure rise at the beam injection, initially observed at intensity above a certain intensity threshold $\left(\mathrm{I}_{\mathrm{th}}\right)$, which was very sensitive to bunch intensity and spacing, and tended to approach saturation at constant intensity. It seems that the electron cloud is responsible for this type of pressure rise [5]. It has limited the $\mathrm{Au}$ intensity for 55-bunch injection, and prevented the 110-bunch operation. The second type of pressure rise occurred at the transition, somewhat proportional to the total beam intensity, and not sensitive to the bunch intensity and spacing. This type of pressure rise has not limited the beam intensity. However, it has caused high detector background and hampers further luminosity improvement.

More warm sections were in-situ baked during the 2002 shutdown with the goal to reduce secondary electron yield (SEY) and molecular desorption yield, and the resulting pressure rise, and to increase $\mathrm{I}_{\text {th }}$. The loosely bound surface contaminants have a much higher SEY and can be

\footnotetext{
*Work performed under Contract Number DE-AC02-98CH10886 with the auspices of the US Department of Energy

†hseuh@bnl.gov
} 
removed through in-situ bake. The intensity thresholds of the $1^{\text {st }}$ type of pressure rise can be obtained by analyzing the pressure rise patterns under various running conditions [3]. The effectiveness of the bakeout for increasing $I_{t h}$ is illustrated in Fig. 2 where the pressure rise at IP12 is compared with proton beam intensity before (2001-2002) and after (2003) bake. For the same increase in pressure, the intensity thresholds were about a factor of two higher after bake. The average $I_{\text {th }}$ values for warm sections at IP12 for various running modes are summarized in Table 1. $I_{\text {th }}$ for $\mathrm{p}$ and $\mathrm{d}$ are much higher than $\mathrm{Au}$, even after factoring in the total charges. $\mathrm{I}_{\text {th }}$ increased by $50 \%$ or more after in-situ bake.
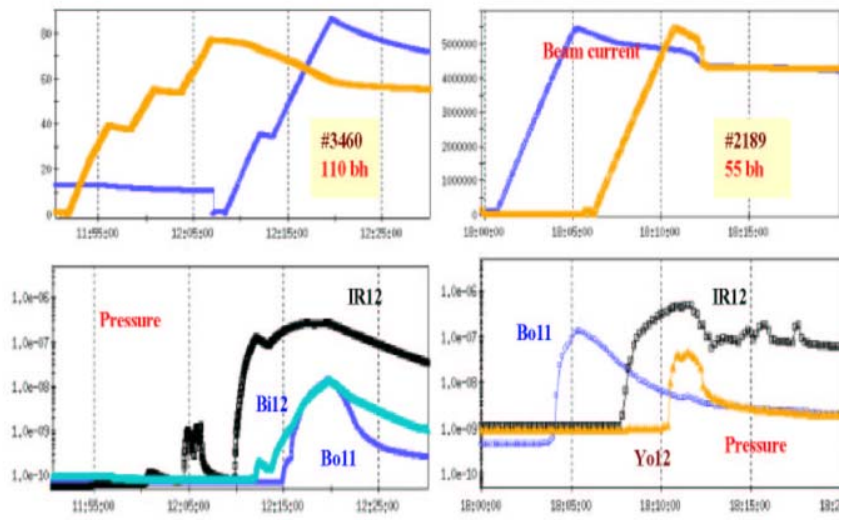

Fig. 2 Comparison of pressure rise with intensity at IP12 with 55- (2002, before bake, in $10^{6}$ unit) and 110-bunch (2003, after bake, in $10^{11}$ unit) protons. The pressure rise is smaller while the intensity is $\sim 50 \%$ higher after bake.

Table 1. $\mathrm{I}_{\text {th }}$ for different species and running modes at IP12

\begin{tabular}{|l|l|l|l|}
\hline & \multicolumn{1}{|c|}{ IP } & Single & comment \\
\hline $\mathrm{p}-55$ & $7 \times 10^{12}$ & $4 \times 10^{12}$ & $2002-$ not baked \\
\hline $\mathrm{p}-110$ & $6 \times 10^{12}$ & $2 \times 10^{12}$ & $2002-$ not baked \\
\hline $\mathrm{Au}^{+79}-55$ & $7 \times 10^{10}$ & $2 \times 10^{10}$ & $2001-$ not baked \\
\hline $\mathrm{Au}^{+79}-110$ & $\sim 6 \times 10^{10}$ & $1 \times 10^{10}$ & $2001-$ not baked \\
\hline $\mathrm{Au}^{+79}-55$ & $>12 \times 10^{10}$ & $4.5 \times 10^{10}$ & $2003-$ baked \\
\hline $\mathrm{Au}^{+79}-110$ & & $3.5 \times 10^{10}$ & $2003-$ baked \\
\hline $\mathrm{d}-55$ & $>12 \times 10^{10}$ & $>7 \times 10^{12}$ & $2003-$ baked \\
\hline $\mathrm{d}-110$ & & $7 \times 10^{12}$ & $2003-$ baked \\
\hline $\mathrm{p}-110$ & $9 \times 10^{12}$ & $3.5 \times 10^{12}$ & $2003-$ baked \\
\hline
\end{tabular}

\section{Electron Detectors and Solenoids}

Eleven custom electron detectors (ED) as shown in Fig. 3 , were designed, fabricated and installed during the 2002 shut down. They were modeled after ED used at Argonne and CERN, but include an additional shield to reduce $\mathrm{rf}$ noise from the beam image current. The bias voltage of the grids can be varied to measure the energy spectrum of the electrons or ions. The transmission efficiency of the ED has been calibrated up to $1500 \mathrm{eV}$ using an electron gun, and found to be around 10\% [6].

Beam pipe solenoids were also installed on the $12 \mathrm{~cm}$ beam pipes bracketing EDs, using 10 gage wire and alternating the winding direction every meter. The solenoid and the DC power supply provide up to 6000 Ampere turn per meter, equivalent to a 75 gauss axial field, which is sufficient to confine electrons of $300 \mathrm{eV}$ (which have peak SEY values) in spiral orbits of $<1 \mathrm{~cm}$ in radius. The solenoids have been energized during the machine studies in 2003. Significant reduction in both the electron signal and the pressure rise has been observed [5, 7].

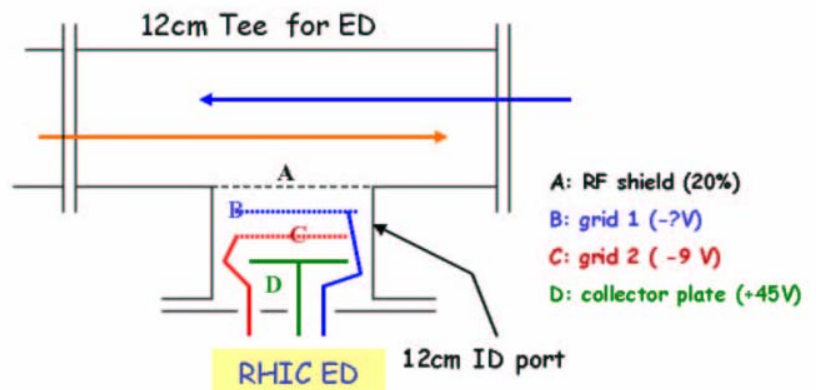

Fig. 3 RHIC electron detectors (ED) consist of an RF shield, two grids and a collector, and have an effective area of over $100 \mathrm{~cm}^{2}$

\section{Vacuum Monitoring and Interlock}

During the 2001-2002 physics runs, the beam was aborted by the vacuum beam permit in a dozen cases. Among them, a few were caused by electrical noise that induced spikes in the vacuum gauge readings used for valve interlock through programmable logic controllers (PLC). These spikes would last a few seconds at most. Prior to the 2003 run, the PLC ladder logic software was modified to include a time delay for triggering the valve closures and beam aborts. No premature triggering has been recorded since. The time delay has also helped preserve beam stores during injection and transition, when pressure would rapidly rise and fall.

The monitoring and control of RHIC vacuum devices is through the use of RS485 serial communication. Up to 32 like devices are connected to a common PLC coprocessor port. Due to the integral processing time of the co-processors, the vacuum readings are updated every 8 seconds for vacuum gauges, and about 30 seconds for devices like partial pressure analyzers and turbopump stations. To monitor and correlate the pressure rise with the beam intensity and other machine parameters, faster data logging was implemented during 2002 shutdown. The analog output signals from selected CCGs around the ring were fed into MADC modules, digitized and logged at a typical rate of $1 \mathrm{~Hz}$. The enhanced data logging capability allows accurate comparison and correlation of the pressure rise with the signals from beam current monitors, ED and beam loss monitors.

\section{FUTURE IMPROVEMENT}

To meet the physics goals in RHIC for year 2004 and beyond, the total beam intensity has to be increased by a factor of two or more, either through increase in bunch intensity, number of bunches or both. The expected pressure rise during injection and transition will be excessive based on observation from the past years. The following remedies are being evaluated and/or implemented to reduce the pressure rise. 


\section{Bakeout of Outgoing Beam Lines}

The major goal in the last few years was to lower the pressure at the incoming insertion beam lines and at the common beam lines around the detectors, thus reduce the detector background to the major experiments. However, during a few high intensity stores, excessive pressure rise was observed at the outgoing lines where most diagnostic equipments with high outgassing components were located. This high pressure has caused large amount of beam scattering and beam loss in the long arcs, which might have induced magnet quenches in the arcs. Therefore these outgoing sections will be in-situ baked to the maximum allowable temperature to reduce the static and dynamic pressure. Non-conventional bakeout methods will be developed for some of these sections.

\section{Beam Tube Solenoids}

Approximately $60 \mathrm{~m}$ of beam tube solenoids were installed and operational for the 2003 run. Significant reduction in ED signals and pressure was observed when solenoids bracketing the ED were energized during machine studies. The present solenoid cables have PVC jackets and have to be removed prior to in-situ bakeout. Kapton insulated wire will be installed for additional solenoids for its radiation and temperature properties.

\section{Collimators}

The existing collimators, one in each ring, are not effective in removing the beam halos and had at times increased backgrounds to the detectors of nearby experiment. These two collimators will be relocated to IR12 region where no major experiment exists. Additional secondary collimators and second-secondary collimators will be installed downstream of the relocated primary collimators to stop secondary particles. By intercepting the beam halos and secondary particles at normal incidence, the molecular desorption rate is expected to be a few decades less than the estimate $10^{7}$ desorbed molecules per ion at grazing angle [3]. The collimators will also be in-situ baked to reduce the magnitude of pressure rise during their operation.

\section{NEG Coating and Lumped Pumps}

Due to large pump spacing and the small linear conductance of the RHIC beam tubes, the effective pumping speed is in tens $1 / \mathrm{s} . \mathrm{m}$ range for $\mathrm{H}_{2}$ and a few 1/s.m for CO. To increase the effective pumping speed, additional lumped pumps or linearly distributed pumps are needed in the warm sections. Lumped pumps in the form of TSP will be effective in the insertion regions, perhaps midway between the existing IP and TSP. It will increase the effective pumping speed by a factor three or more. Linearly distributed pumps in the form of nonevaporable getter (NEG) coating [8] will be more effective in the interaction regions where little transverse space is available for lumped pumps and the linear conductance is smaller than that of insertion regions. The NEG coating will provide effective pumping speed of $10^{2}$ 1/s.m and will also reduce the SEY, and the electron and ion desorption rates. The coating of the existing $7 \mathrm{~cm} \Phi$ beryllium beam tubes is certainly challenging if not impossible. The addition of high $\mathrm{Z}$ material on the detector beam tubes has to be evaluated by the experimenters for its reduced transparency to the energetic particles. The periodic activation requirement of NEG coating has to be incorporated into vacuum system operation. A few NEG coated beam tubes will be installed at one warm section and evaluated during the upcoming run.

\section{SUMMARY}

Improvement of the RHIC vacuum system before 2003 physics runs has produced some positive effect on the beam intensity thresholds for the pressure rise as compared with those of the 2001 and 2002 runs. The magnitude of the pressure rise is also lower than that of the previous years. Solid evidence of electron cloud from the newly-installed electron detectors was seen during high intensity runs. The effect of the solenoids to suppress the electron activities has been observed. Further improvement is needed to allow increase in intensity. To that end, more warm sections will be in-situ baked and additional collimators will be installed to intercept the beam halo at normal incidence. Additional lumped pumps and NEG coating will also increase the effective pumping speed several-fold.

\section{ACKNOWLEDGEMENTS}

The authors would like to thank M. Blaskiewicz, W. Fischer, U. Iriso-Ariz and D. Trbojevic of BNL for fruitful discussion and members of Collider-Accelerator Vacuum Group for their effort in improving the RHIC vacuum systems.

\section{REFERENCES}

[1] M.J. Rhoades-Brown and M. A. Harrison, RHIC Technical Note \#106, BNL-47070, Dec. 1993.

[2] H.C. Hseuh, et al, Proc. PAC'99, New York, 557 (1999).

[3] H.C. Hseuh, L.A. Smart and S.Y. Zhang, Proc. EPAC'02, Paris, 2559 (2002).

[4] W. Fischer, et al, Proc. EPAC'02, Paris, 1485 (2002).

[5] S.Y. Zhang, et al, 'RHIC Pressure Rise and Electron Cloud', These Proceedings.

[6] P. He, et al, 'Calibration of RHIC Electron Detectors', These Proceedings.

[7] U. Iriso-Ariz, et al, 'Electron Detectors for Vacuum Pressure Rise Diagnostics at RHIC', These Proceedings.

[8] C. Benvenuti, Proc. PAC'01, Chicago, 602 (2001). 